CONSTRUCTION DE SUPPORTS DE PLANTATION FERTILES A PARTIR DE MOUSSES DE POLYURETHANE RECYCLEES

\title{
CONSTRUCTION OF FERTILE GROWING MEDIA FROM RECYCLED POLYURETHANE FOAM
}

\author{
ARTOUS Mathieu ${ }^{1}$, GUÉNON René ${ }^{1}$, LEMMEL Olivier², BUORD Hervé ${ }^{3}$, VIDAL-BEAUDET Laure ${ }^{1}$, CANNAVO Patrice ${ }^{1 *}$ \\ ${ }^{1}$ Institut Agro, EPHOR, 49000 Angers, France \\ 2 Brangeon Recyclage, 4 rue Chevreul, 49300 Cholet \\ 3 Jaulin Paysages, Chemin des Gruellières, 44470 Carquefou
}

*Auteur correspondant : patrice.cannavo@agrocampus-ouest.fr

\section{RESUME}

Inventé dans les années 1930, les polyuréthanes (PU) sont un groupe spécial de matériaux polymères. En France, seulement $3 \mathrm{kt}$ de PU sont recyclés annuellement sur une production de $250 \mathrm{kt}$. Le potentiel de valorisation de ce déchet est donc questionné et la voie agronomique pour la construction de supports de cultures non alimentaire pourrait être envisagée.

Une étude a été menée pour évaluer la pertinence d'apport de mousses PU dans un substrat de plantation, afin de savoir si elles peuvent lui conférer des caractéristiques physico-chimiques intéressantes pour la croissance végétale sans risque pour l'environnement.

Les substrats testés sont des mélanges de compost (Matière d'Intérêt Agronomique issue du Traitement des Eaux, NF U44-095), de mousses PU et de terre végétale. Les mousses utilisées sont issues de l'âme de matelas en PU. Quatre ratios volumiques de mélanges compost/mousse ont été réalisés. De la terre végétale a été ajoutée à différents ratios volumiques pour constituer les substrats finaux (16 substrats finaux).

Les analyses écotoxicologiques montrent l'innocuité environnementale des substrats testés. Des essais sous serre pendant 54 jours ont permis de suivre le développement du ray-grass dans les substrats. Des supports de plantation avec un potentiel agronomique adapté pour les toitures végétalisées ont été identifiés, sur la base des résultats expérimentaux de l'étude, et seront testés à plus grande échelle.

MOTS-CLES : Compost, sol arable, ray grass, propriétés agronomiques, innocuité environnementale, toiture végétalisée

\begin{abstract}
Invented in the 1930s, polyurethanes (PU) are a special group of polymeric materials. In France, only $3 \mathrm{kt}$ of PU are recycled annually out of a production of $250 \mathrm{kt}$. The potential of recovery of this waste is therefore questioned and the agronomic way for the construction of fertile soils is considered.
\end{abstract}

A study was conducted to evaluate the relevance of adding PU foam in a growing media (GM) for plantation, and to know if they can confer interesting characteristics for plant growth without any risk for the environment.

The tested GMs were mixtures of compost (Material of Agronomic Interest from Water Treatment, NF U44-095), foams and arable topsoil. The foams used were derived from the core of PU mats. Four volume ratios of compost/foam mixtures were achieved. Topsoil was added at different volume ratios to constitute the final GMs (16 final GM).

Ecotoxicological analyses show the safety of the tested GMs. Greenhouse trials over 54 days allowed to follow the development of ryegrass in GMs. Planting media with suitable agronomic potential for green roofs have been identified, based on the experimental results of the study, and will be tested on a larger scale.

KEYWORDS: compost, arable soil, rye grass, agronomic properties, environmental safety, green roof 


\section{INTRODUCTION}

La population mondiale devrait atteindre 9,1 milliards d'habitants d'ici à 2050, soit $34 \%$ de plus qu'en 2017 (United Nations 2018). Cette augmentation se traduit par une urbanisation croissante, et les mégalopoles constituent un enjeu environnemental préoccupant (i.e. pollution, ilot de chaleur urbain, etc...) (Levin et al. 2017). Conjointement à cette problématique, la demande sociétale montre un besoin de végétalisation des villes pour pallier les conditions de vie qui vont se dégrader avec le changement climatique. Les solutions fondées sur la nature constituent un levier d'action pour garantir des bénéfices naturels tels que la protection des sols, la réduction des émissions de polluants, la régulation thermique, la qualité de l'air et la biodiversité.

L'usage de sol de décapage issu des terres agricoles pour la mise en place d'espaces verts urbains fait aujourd'hui polémique en raison de la diminution des surfaces cultivables. Damas et Coulon (2016) ont estimé que 3 millions de mètres cube de terre, venant essentiellement des sols agricoles, sont nécessaires chaque année en France pour la mise en place $d$ 'espaces végétalisés urbains. Ainsi, la réutilisation de déchets ou de sous-produits pour construire des sols remplissant leurs fonctions attendues peut être une alternative à la protection des ressources naturelles (Séré et al. 2008). Inventé en Allemagne dans les années 1930, le polyuréthane (PU) est un matériau issu de l'industrie chimique, constitué de polymères très différents des autres matériaux plastiques. Le PU peut être incorporé dans différents produits, tels que peinture, revêtement liquide, élastomères, isolant, fibres élastiques, mousses, etc... (Boujard et al., 2014). En France, le gisement annuel de déchets PU est estimé à $210 \mathrm{kt}$ et seulement $3 \mathrm{kt}$ sont recyclées chaque année (Boujard et al., 2014). Des filières de valorisation de ces mousses sont donc recherchées. Parmi celles-ci, la valorisation agronomique de ces matériaux dans des supports de plantation pourrait être envisagée. Les mousses de polyuréthane présentent l'avantage d'avoir une très faible densité (en moyenne entre 20 et $30 \mathrm{~kg} \cdot \mathrm{m}^{-3}$ ) (Boujard et al., 2014), permettant de concevoir des substrats de plantation légers qui seraient compatibles, par exemple, avec les contraintes d'aménagement de toitures végétalisées. Néanmoins, à notre connaissance, aucune étude n'a porté sur l'intégration de mousses de polyuréthane dans des sols ou substrats servant de supports de culture.

Ainsi, la valorisation agronomique pourrait être une voie potentielle de recyclage du polyuréthane en développant des supports de cultures pour les plantes à vocation ornementale. L'objectif de ce travail est d'évaluer l'aptitude de différents substrats de plantation à base de polyuréthane à favoriser la croissance des plantes tout en garantissant l'innocuité environnementale à l'aide d'analyses écotoxicologiques (ETM, HAP, PCB, COV, agents ignifugeants, micro-organismes et agents pathogènes). Les substrats testés sont des mélanges de compost, de terre végétale et de mousses de polyuréthane.

\section{MATERIELS ET METHODES}

\subsection{Préparation des mousses}

Les mousses utilisées dans le cadre de cette étude sont issues de l'âme de matelas en polyuréthane (le gisement annuel est d'environ 49 kt en France). Ces matelas proviennent d'un gisement établi par Ecomobilier, éco-organisme à but non lucratif, créé en décembre 2011 pour la collecte et le recyclage du mobilier usagé en France. Ces matelas ont pu servir à une multitude d'utilisations. L'entreprise Brangeon Recyclage, Cholet (France), a réceptionné en 2019 un chargement de mousses représentatif du gisement global. $200 \mathrm{~m}^{3}$ ont été broyés sur place (environ $20 \mathrm{t}$ ) en deux étapes: (1) un pré-broyage grossier permettant de préparer les mousses et (2) un broyage final pour obtenir une granulométrie de $20 \mathrm{~mm}$.

\subsection{Préparation des mélanges}

Afin de créer et d'évaluer la valeur agronomique des supports de plantation à base de mousses de polyuréthane, les mousses ont été associées à deux autres matériaux : du compost et de la terre végétale. Pour favoriser l'assemblage des mousses de faible masse volumique apparente avec les autres matériaux, les mélanges ont été réalisés en deux temps. Dans un premier temps, un mélange mousse de PU - compost a été élaboré et réalisé par l'entreprise Brangeon Recyclage. Le compost utilisé pour effectuer les mélanges est un compost normé de Matières d'Intérêt Agronomique issues du Traitement des Eaux (NFU 44-095) constitué de 2 volumes de refus de crible, 1 volume de végétaux et 1 volume de boues d'épuration. Les mousses ont été intégrées au processus de compostage (au cours de la phase de fermentation) afin de subir une hygiénisation par vapeur d'eau. A l'issue de ce compostage, quatre ratios volumiques de mélanges compost-mousse ont été produits et maturés durant 9 semaines (aération forcée, maturation et criblage de la matière). Ces mélanges volumiques compost-mousse sont notés : M1 (100:0); M2 (80:20); M3 (60:40); M4 (40:60). Dans un deuxième temps, les mélanges $M 1, M 2, M 3$ et $M 4$ ont été assemblés avec de la terre végétale qui provient du site de l'Institut Agro Rennes-Angers (France). Cette terre végétale nommée TV provient 
du décapage de l'horizon 0-20 $\mathrm{cm}$ d'une parcelle agricole (texture sablo-limoneuse). Différents volumes de terre arable : $0 \%, 20 \%, 40 \%$ et $60 \%$ (respectivement nommés TV0, TV20, TV40 et TV60) ont été apportés aux mélanges compost-mousse.
Ainsi, 16 modalités ont été constituées afin d'évaluer leurs propriétés agronomiques (Tableau 1 ).

Tableau 1 : Modalités testées durant l'expérimentation issues de l'assemblage des mélanges compost-mousse M1, M2, M3, M4 et de terre végétale

\begin{tabular}{ccccc}
\hline & $\begin{array}{c}100 \% \text { Compost }- \\
0 \% \text { Mousses } \\
\text { M1 }\end{array}$ & $\begin{array}{c}80 \% \text { Compost }- \\
20 \% \text { Mousses } \\
\text { M2 }\end{array}$ & $\begin{array}{c}60 \% \text { Compost }- \\
40 \text { Mousses } \\
\text { M3 }\end{array}$ & $\begin{array}{c}40 \% \text { Compost - } \\
60 \text { Mousses } \\
\text { M4 }\end{array}$ \\
\hline $\begin{array}{c}100 \% \text { du mélange } \\
+0 \% \text { terre végétale }\end{array}$ & M1-TV0 & M2-TV0 & M3-TV0 & M4-TV0 \\
\hline $\begin{array}{c}80 \% \text { du mélange } \\
+20 \% \text { terre végétale }\end{array}$ & M1-TV20 & M2-TV20 & M3-TV20 & M4-TV20 \\
\hline $\begin{array}{c}60 \% \text { du mélange } \\
+40 \% \text { terre végétale }\end{array}$ & M1-TV40 & M2-TV40 & M3-TV40 & M4-TV40 \\
\hline $\begin{array}{r}40 \% \text { du mélange } \\
+60 \% \text { terre végétale }\end{array}$ & M1-TV60 & M2-TV60 & M3-TV60 & M4-TV60 \\
\hline
\end{tabular}

\subsection{Préparation de l'essai}

L'essai a été réalisé à l'Institut Agro Rennes-Angers $\left(47^{\circ} 28^{\prime} \mathrm{N}, 0^{\circ} 33^{\prime} \mathrm{E}\right)$ dans un compartiment de serre en verre entre mai et juillet 2019. Le climat a été contrôlé automatiquement; la température de l'air a été maintenue à $22^{\circ} \mathrm{C}\left( \pm 2{ }^{\circ} \mathrm{C}\right)$ pendant le jour $(16 \mathrm{~h} /$ jour $)$ et $18{ }^{\circ} \mathrm{C}\left( \pm 2{ }^{\circ} \mathrm{C}\right)$ pendant la nuit $(8 \mathrm{~h} /$ jour $)$ par ventilation grâce à des ouvrants sur le toit de la serre et des écrans d'ombrage au-dessus de la canopée.

Les masses volumiques ainsi que les humidités des quatre mélanges $M 1, M 2, M 3$ et $M 4$, et de la terre végétale ont été déterminées afin de calculer les quantités de chaque matériau à intégrer dans les pots (ISO 11272:2017). Les pots utilisés durant l'essai avaient un volume de $3 \mathrm{~L}$. L'espèce végétale sélectionnée est le Ray-grass anglais (Lolium perenne) car cette plante fourragère est utilisée seule ou en mélange dans différents types d'espaces verts mais aussi en végétalisation de sites dégradés et/ou pollués ou de sols construits (Cordova et al. 2011). Le Ray-grass a été semé le 20 mai 2019 à raison de 5 graines par pot, soit une densité de $200 \mathrm{~kg}$.ha ${ }^{-1}$ ) et récolté le 12 juillet 2019.

Six répétitions par modalité ont été mises en place, pour un total de 96 pots disposés sur une tablette. La répartition des pots s'est faite selon un schéma aléatoire. Des pots ont également été disposés en bordure de l'essai pour limiter les effets de bord. L'arrosage de la tablette était automatique et a été fait quotidiennement par sub-irrigation (tous les jours à $10 \mathrm{~h}$, remplissage pendant 5 minutes et mouillage pendant 20 minutes).

\subsection{Analyses réalisées}

\subsubsection{Analyse initiale des matériaux}

Chaque mélange compost-mousse (M1, M2, M3, M4) a été analysé afin de vérifier la conformité de ces substrats avec la norme support de culture (NF U44551). Des analyses complémentaires ont été réalisées avec deux principaux objectifs : (1) mettre en évidence l'innocuité environnementale de ces substrats en dosant les composés organiques volatils (COV) (méthode interne - Laboratoire Inovalys) ; les PCB et HAP (méthode interne selon NF EN 16167 et XP X33-012) ; les diphényléthers polybromés (BDE), et (2) avoir une connaissance des propriétés physicochimiques des substrats en déterminant les courbes de rétention en eau (NF U44-175).

\subsubsection{Production de biomasse}

A la fin de l'expérimentation, la biomasse aérienne a été récoltée puis pesée fraîche et sèche. Le ray-grass a été séché à $60^{\circ} \mathrm{C}$ pendant $48 \mathrm{~h}$ à l'étuve puis pesé, à raison de 6 répétitions par modalité.

\subsection{Traitements statistiques}

Nous avons étudié les effets des différentes modalités compost-mousse et terre végétale selon une ANOVA à 2 facteurs $(p<0,05)$ sur les propriétés physicochimiques des différents mélanges et sur la biomasse aérienne de Ray-Grass, grâce au logiciel Statistica 13.0. Parmi les modalités, des comparaisons de moyenne entre les biomasses aériennes ont été évaluées avec le test post hoc HSD (honestly significant difference) de Tukey à $p<0,05$. Une analyse en composantes principales (ACP) a été réalisée avec le package FactoMineR (Lê et al, 2008). 


\section{RESULTATS}

\subsection{Caractéristiques initiales des matériaux}

Les analyses écotoxicologiques des mélanges compost-mousse ont montré que les mélanges $M 1$, M2, M3 et M4 présentent des valeurs microbiologiques, de HAP, PCB, COV et ETM, toutes en-dessous des seuils limites exigés par la norme NF U44-551 ou en-dessous du seuil de détection (résultats non présentés). Les limites de détection sont bien inférieures aux seuils exigés par la norme. Le Tableau 2 présente les résultats des analyses physico-chimiques des mélanges compost-mousse et de la terre végétale.

Tableau 2 : Paramètres physico-chimiques de la terre végétale TV et des mélanges M1, M2, M3 et M4. Les seuils de préconisations indiqués dans la dernière colonne correspondent à des recommandations pour des sols et substrats. Les valeurs en gras mentionnent une valeur excédentaire ou déficitaire au regard de la recommandation

\begin{tabular}{|c|c|c|c|c|c|c|c|}
\hline \multirow{2}{*}{ Paramètre } & \multicolumn{5}{|c|}{ Matériau / Mélange } & \multirow{2}{*}{ Unité } & \multirow[b]{2}{*}{ Préconisation } \\
\hline & M1 & M2 & M3 & $\mathrm{M} 4$ & TV & & \\
\hline $\mathrm{pH}$ & 9,1 & 6,53 & 6,75 & 7,06 & 7,70 & - & $\begin{array}{l}6,5 \text { à } 7,5 \text { (Damas et } \\
\text { Coulon, 2016) }\end{array}$ \\
\hline Conductivité & 9,1 & 2,66 & 2,15 & 2,6 & 0,10 & $\mathrm{mS} . \mathrm{cm}^{-1}$ & $<0,5$ \\
\hline $\begin{array}{c}\text { Matières organiques } \\
\text { à } 450^{\circ} \mathrm{C}\end{array}$ & 44,6 & 58,0 & 55,7 & 63,7 & 5,04 & g. $100 \mathrm{~g}^{-1} \mathrm{MS}$ & - \\
\hline Matières sèches & 58,9 & 60,1 & 57,1 & 60,7 & 92,5 & g. $100 \mathrm{~g}^{-1} \mathrm{MS}$ & - \\
\hline Azote total & 11,80 & 13,92 & 15,91 & 18,04 & 2,60 & gN.kg-1 MS & 10 à 20 (Damas et \\
\hline Azote ammoniacal & 1,5 & 2,31 & 2,24 & 2,81 & $<0,10$ & $\mathrm{gN} \cdot \mathrm{kg}^{-1} \mathrm{MS}$ & - \\
\hline Nitrates & 2300 & 642,6 & 495,2 & 338,9 & 52,0 & $\mathrm{mg} \cdot \mathrm{kg}^{-1} \mathrm{MS}$ & - \\
\hline Bore (B) & 16,9 & 21,05 & 19,40 & 19,40 & 0,37 & $\mathrm{mg} \cdot \mathrm{kg}^{-1} \mathrm{MS}$ & $\begin{array}{l}\text { 0,10 à 0,40 (Wiki } \\
\text { Auréa, 2014) }\end{array}$ \\
\hline Cobalt (Co) & 5,5 & 3,03 & 2,80 & 2,65 & 15 & $\mathrm{mg} \cdot \mathrm{kg}^{-1} \mathrm{MS}$ & \\
\hline Fer (Fe) & 11594,7 & 10362,6 & 9134,8 & 10788,7 & 86,80 & $\mathrm{mg} \cdot \mathrm{kg}^{-1} \mathrm{MS}$ & $\begin{array}{c}10 \text { à } 300 \text { (Becker et } \\
\text { Asch, 2005) }\end{array}$ \\
\hline Manganèse (Mn) & 192,9 & 262,6 & 248,9 & 203,4 & 21,20 & $\mathrm{mg} \cdot \mathrm{kg}^{-1} \mathrm{MS}$ & $\begin{array}{c}10 \text { à } 100 \text { (Gobat et al, } \\
2010 \text { ) }\end{array}$ \\
\hline Molybdène (Mo) & 1,20 & 1,10 & 0,90 & 1,20 & 0,50 & $\mathrm{mg} \cdot \mathrm{kg}^{-1} \mathrm{MS}$ & $>0,15$ (Grigg, 1953) \\
\hline $\begin{array}{l}\mathrm{CaO} \text { échangeable } \\
\mathrm{K}_{2} \mathrm{O} \text { échangeable } \\
\mathrm{MgO} \text { échangeable }\end{array}$ & $\begin{array}{c}121,69 \\
16,17 \\
7,75\end{array}$ & $\begin{array}{c}23,89 \\
14,51 \\
4,59\end{array}$ & $\begin{array}{c}24,05 \\
14,93 \\
4,66\end{array}$ & $\begin{array}{l}20,33 \\
10,60 \\
\mathbf{3}, 65\end{array}$ & $\begin{array}{l}7,15 \\
0,52 \\
\mathbf{0 , 3 9}\end{array}$ & $\begin{array}{l}\text { g. } \mathrm{kg}^{-1} \mathrm{MS} \\
\text { g. } \mathrm{kg}^{-1} \mathrm{MS} \\
\mathrm{g} . \mathrm{kg}^{-1} \mathrm{MS}\end{array}$ & $\begin{array}{l}- \\
- \\
-\end{array}$ \\
\hline $\mathrm{Na}_{2} \mathrm{O}$ échangeable & 1,28 & 0,095 & 0,098 & 0,070 & 0,085 & g. $\mathrm{kg}^{-1} \mathrm{MS}$ & - \\
\hline $\begin{array}{l}\text { Capacité d'Echange } \\
\text { Cationique (CEC) }\end{array}$ & 54,44 & 44,26 & 41,22 & 31,14 & 9,89 & meq. $100 \mathrm{~g}^{-1} \mathrm{MS}$ & $\begin{array}{l}>40 \text { (Damas et } \\
\text { Coulon, 2016) }\end{array}$ \\
\hline $\mathrm{P}_{2} \mathrm{O}_{5}$ Olsen & 0,71 & 1,25 & 1,53 & 1,06 & 0,16 & g. $\mathrm{kg}^{-1}$ de MS & $\begin{array}{l}>0,12 \text { (Damas et } \\
\text { Coulon, 2016) }\end{array}$ \\
\hline $\begin{array}{l}\text { Masse volumique } \\
\text { apparente sèche }\end{array}$ & 0,44 & 0,23 & 0,20 & 0,16 & 1,28 & g. $\mathrm{cm}^{-3}$ & $\begin{array}{c}<1,2 \text { (Damas et } \\
\text { Coulon, 2016) }\end{array}$ \\
\hline Porosité totale & 79,94 & 87,48 & 88,71 & 90,53 & 49,72 & $\%$ vol & \\
\hline Macroporosité & 39,04 & 48,68 & 52,01 & 65,73 & 5,30 & $\%$ vol & $\begin{array}{c}>20 \%(\text { Damas et } \\
\text { Coulon, 2016) }\end{array}$ \\
\hline Réserve utile & 0,89 & 0,72 & 0,52 & 0,62 & 1,07 & $\mathrm{~mm} \cdot \mathrm{cm}^{-1}$ & $\begin{array}{c}>1 \text { (Damas et Coulon, } \\
2016)\end{array}$ \\
\hline $\begin{array}{l}\text { Capacité de } \\
\text { rétention en eau } \\
\text { (à-10kPa) }\end{array}$ & 0,500 & 0,388 & 0,367 & 0,248 & 0,444 & $\%$ vol & - \\
\hline
\end{tabular}

Le compost seul (M1) présente un $\mathrm{pH}$ basique $(9,1)$, une conductivité électrique élevée $(9,1 \mathrm{mS} / \mathrm{cm})$, une forte concentration en nitrates, des concentrations élevées en bore, fer, manganèse et magnésium. L'ajout de mousses à différentes proportions au compost seul permet de modifier favorablement ses caractéristiques chimiques initiales. Concernant la terre végétale, les teneurs en éléments nutritifs sont bien inférieures à celles contenues dans le compost seul et les autres mélanges avec les mousses. Sa qualité agronomique est globalement satisfaisante, malgré un $\mathrm{pH}$ un peu élevé, une teneur en $\mathrm{Mg}$ échangeable et une capacité d'échange cationique (CEC) plutôt faibles.

Les analyses physiques des mélanges compostmousses, montrent pour l'ensemble des mélanges une masse volumique apparente intéressante pour un substrat de plantation et, par conséquent une porosité totale élevée (> à $80 \%$, Tableau 2). Leur macroporosité est également importante (> à $39 \%$ ). Seule la réserve utile est légèrement inférieure aux 
préconisations. Si on s'intéresse plus spécifiquement aux mélanges, l'ajout de mousses à différentes proportions au compost pur modifie sensiblement ses caractéristiques initiales. Elles allègent le mélange (masse volumique apparente dans M4 est divisée par 2,8 par rapport à $\mathrm{M} 1$ ). Cette baisse de masse volumique conduit à une augmentation de la porosité totale, qui se traduit par une augmentation de la macroporosité (qui a presque doublé entre M1 et M4), et par une baisse de la microporosité représentée par la réserve utile. La terre végétale quant à elle est plus dense que les mélanges compostmousse, 2,9 fois plus que le mélange $M 1$. Sa réserve utile est satisfaisante, mais sa macroporosité relativement faible.

\subsection{Croissance du ray-grass anglais}

La production de biomasse de ray-grass en fonction des modalités est présentée sur la Figure 1 . Les 2 facteurs TV (F-value 129, $\mathrm{p}<0.001$ ) et M (F-value 2.9, $p<0.05$ ) ont un effet significatif sur la croissance du Ray-grass et l'interaction entre ces deux facteurs a également un effet significatif ( $F$-value 4.6, $p<0.001$ ).

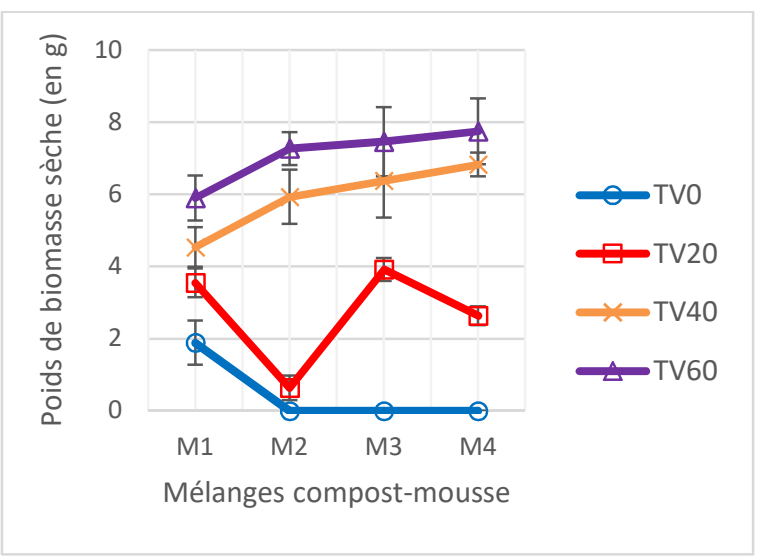

Figure 1 : Biomasse aérienne sèche du ray-grass en fonction de la proportion de mousse dans les mélanges $M$, et selon la proportion de terre végétale $\operatorname{TV}(0,20 \%, 40$ $\%, 60 \%$ en volume)

La quantité de biomasse sèche de ray-grass produite oscille entre 0 et 9 grammes sec en fonction des modalités. Les résultats montrent que pour un mélange $\mathrm{M}$ donné, la biomasse augmente lorsque la teneur en terre végétale augmente. De plus, lorsque I'on a au moins $40 \%$ de TV, plus il y a de mousses plus il y a de biomasse produite (Figure 1). Ainsi, en l'absence de terre végétale (TVO) la croissance des plantes est faible (M1), voire inexistante $(M 2, M 3$, M4). Avec 40 ou $60 \%$ de TV, les biomasses produites sont assez proches quels que soient les mélanges $M$.

L'ACP a permis d'expliquer $86 \%$ de la variance sur les deux premiers axes (figure 2 ). Il existe une corrélation positive selon l'axe 1 entre l'ensemble des teneurs en macro et oligo-éléments et la macroporosité, et une corrélation négative selon le même axe avec la biomasse de ray-grass et la réserve utile. L'axe 2 est corrélé positivement au $\mathrm{pH}$, à la conductivité électrique et aux éléments échangeables $\mathrm{Na}_{2} \mathrm{O}, \mathrm{P}_{2} \mathrm{O}_{5}$ et $\mathrm{MgO}$. L'ACP sépare clairement les individus sans $T V$, à droite et les modalités avec TV, à gauche. Les individus situés en bas à droite (M2, M3 et M4) témoignent de fortes charges en éléments chimiques. Ils correspondent aux mélanges de compost et mousses n'ayant pas produit de biomasse. L'individu M1 situé en haut à droite témoigne également de fortes charges en éléments chimiques mais également d'une valeur de $\mathrm{pH}$ plus élevée. Les individus groupés à gauche correspondent aux modalités avec ajout de terre végétale. Les mélanges ayant produit les quantités de biomasse les plus importantes sont celles les plus à gauche, avec un réservoir d'eau utilisable plus important. L'ajout de terre végétale (de 20 à $60 \%$, soit TV20 à TV60) influe directement sur ce paramètre qui est le seul en corrélation avec la quantité de biomasse aérienne produite.

\section{DISCUSSION}

\subsection{Qualité agronomique des substrats}

Les résultats présentés montrent que sur la base d'un essai de culture de 3 mois, la valorisation agronomique des mousses de polyuréthane est prometteuse, à condition que la préparation des matériaux ajoutés pour remplir la fonction de support de végétation soient bien raisonnée. Toutes les modalités testées ont des caractéristiques conformes à la norme NF U44-551 (support de culture).

L'introduction de mousse dans le compost a eu des effets notables sur les propriétés physico-chimiques du compost seul. Concernant les propriétés physiques, les mélanges sont peu compacts et donc propices au développement racinaire. Les mousses sont des matériaux plus macroporeux que microporeux. Une attention particulière devra être portée à l'arrosage du substrat afin d'assurer le besoin en eau des végétaux.

Les analyses chimiques montrent que si le compost (M1) présente un certain nombre de contraintes agronomiques telles qu'un $\mathrm{pH}$ élevé et des teneurs en macro et oligo-éléments fortes, l'ajout de mousses a une action de dilution de ces valeurs. Ainsi, le pH des mélanges approche la neutralité avec les mousses. En effet, ces dernières libèrent des composés organiques acides au contact de l'eau acidifiant ainsi le milieu (Lovett et Eastop, 2004). 


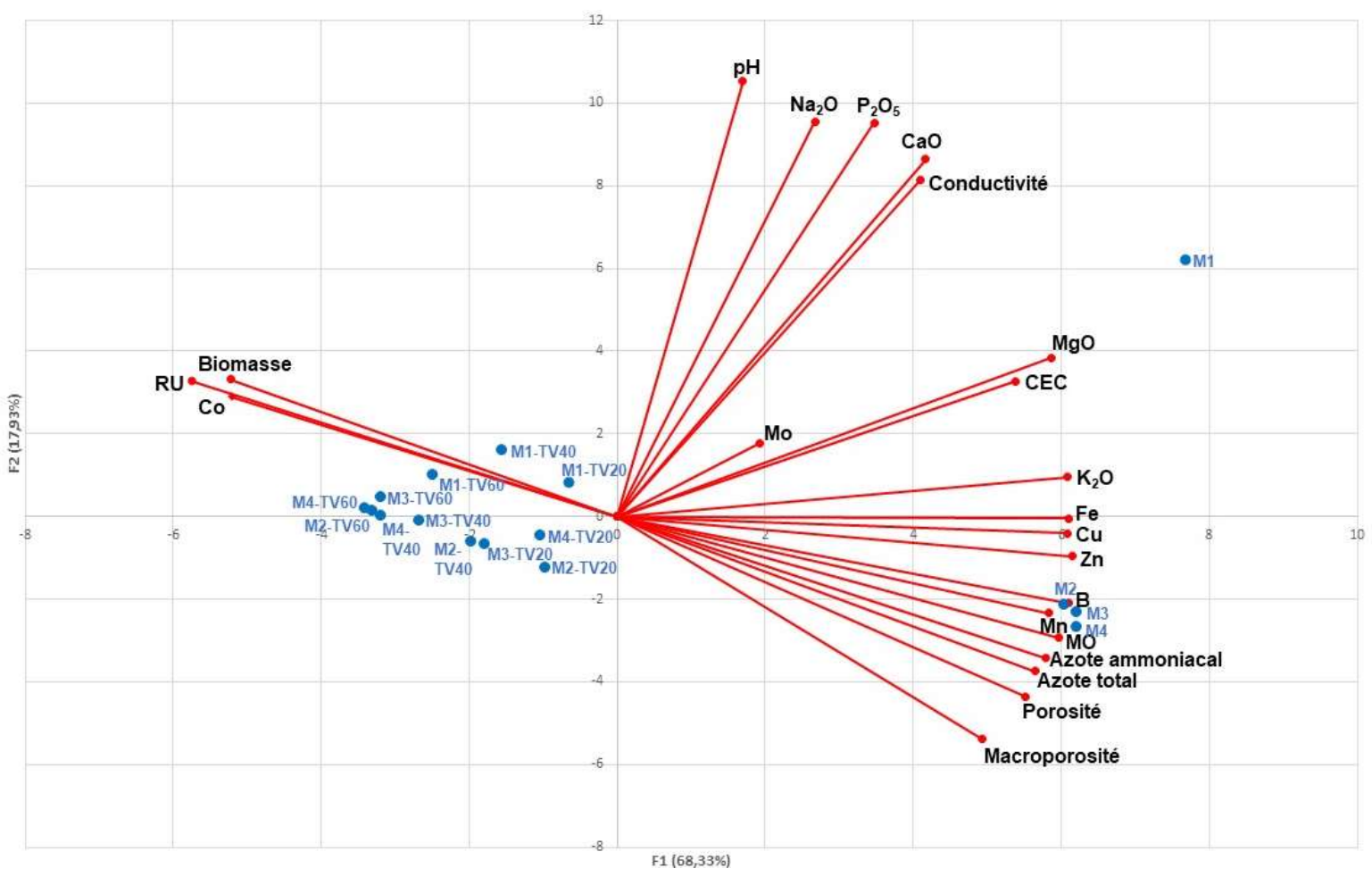

Figure 2 : Analyse en composantes principales des paramètres physico-chimiques et des modalités étudiées

Les teneurs en azote total augmentent également avec l'ajout des mousses, en raison des constituants de ces mousses formées d'isocyanate et d'uréthane qui sont des composés azotés (Pellizzi, 2012). Les nitrates sont une étape du fonctionnement naturel du cycle de l'azote, issus de la minéralisation de l'azote organique. Les fortes teneurs en nitrate présentes dans M1 peuvent être le résultat d'une activité de minéralisation importante pendant toute la durée du compostage, et du stockage du mélange jusqu'à son utilisation. Un risque de lixiviation est donc possible lors des premières précipitations dans le cadre d'un usage en conditions extérieures, et on sait que la gestion des rejets en nitrates dans le milieu naturel est un enjeu majeur. Néanmoins l'ajout de mousses dilue cette concentration (Tableau 2). Le fer est présent en très grande quantité dans les mélanges, susceptible de générer un risque de toxicité pour les plantes (Becker et Asch, 2005).

\subsection{Croissance des plantes, effet de la terre} végétale et usages possibles des substrats

Cette expérimentation a montré que sans terre végétale, la biomasse de ray-grass se développait très difficilement. L'apport progressif de TV (20 à $60 \%$ ) montre une augmentation de la production de biomasse, quel que soit le mélange $M 1, M 2, M 3, M 4$. Cet apport de TV permet d'atténuer les teneurs élevées en éléments nutritifs et en matière organique des mélanges compost-mousse. L'apport de terre permet aussi une meilleure structuration du mélange et donc une amélioration de ses propriétés physiques. Dans l'objectif de valoriser les mousses dans des supports de culture pour des toitures végétalisées, trois types d'usage seraient possibles: les extensif, semi-intensif et intensif. Le facteur limitant pour la mise en place de substrats des toitures végétalisées extensives est la charge exercée par le substrat, qui doit être inférieure à $180 \mathrm{~kg} \cdot \mathrm{m}^{-2}$ pour que la toiture puisse en supporter la charge (ADIVET et al., 2018). Le tableau 4 présente les charges maximales des substrats. Ces calculs ont été effectués à l'aide des valeurs de masses volumiques apparentes sèches mesurées : TV : $1280 \mathrm{~kg} \cdot \mathrm{m}^{-3}$, compost : $440 \mathrm{~kg} \cdot \mathrm{m}^{-3}$, mousse de polyuréthane : $40 \mathrm{~kg} \cdot \mathrm{m}^{-3}$. Les limites de charges sont exprimées en daN $\mathrm{m}^{-2}$ ou bien en $\mathrm{kg} \cdot \mathrm{m}^{-2}$ ( $1 \mathrm{daN}=1 \mathrm{~kg} \pm 2 \%)$. Afin de calculer la charge maximale d'un substrat, il est nécessaire d'appliquer une épaisseur aux masses volumiques apparentes $(15,20$ et $30 \mathrm{~cm}$ ). À cela, il faut ajouter d'autres valeurs telles que: le poids de la couche absorbante et drainante (en moyenne $2 \mathrm{~kg} \cdot \mathrm{m}^{-2}$ ), une charge dite de sécurité (15 kg. $\left.\mathrm{m}^{-2}\right)$, la masse des végétaux (environ $10 \mathrm{~kg} \cdot \mathrm{m}^{-2}$ ) (ADIVET et al., 2018). Sur cette base, les modalités M3-TV20, M4-TV20 et M4-TV40 sont les plus intéressantes, pour des raisons de charge, mais 
surtout pour un potentiel de recyclage de grandes quantités de mousses. Ces substrats sont dans la moyenne haute des substrats potentiellement utilisables en système extensif.

Tableau 4 : Charge exercée par les substrats ayant produit de la biomasse avec une charge maximale en eau. Les valeurs en gras représentent les valeurs admissibles en système extensif $\left(<\right.$ à $\left.180 \mathrm{~kg} \mathrm{~m}^{-2}\right)$

\begin{tabular}{cccc}
\cline { 2 - 4 } & $\begin{array}{c}\text { Charge pour une } \\
\text { épaisseur de } 12 \mathrm{~cm} \\
\left(\mathrm{~kg} \cdot \mathrm{m}^{-2}\right)\end{array}$ & $\begin{array}{c}\text { Charge pour une } \\
\text { épaisseur de } 20 \mathrm{~cm} \\
\left(\mathrm{~kg} \cdot \mathrm{m}^{-2}\right)\end{array}$ & $\begin{array}{c}\text { Charge pour une } \\
\text { épaisseur de } 30 \mathrm{~cm} \\
\left(\mathrm{~kg} \cdot \mathrm{m}^{-2}\right)\end{array}$ \\
\hline M2-TV0 & $\mathbf{6 5}$ & $\mathbf{9 1}$ & $\mathbf{1 2 3}$ \\
M2-TV20 & 114 & 172 & 244 \\
M2-TV40 & 146 & 226 & 326 \\
M2-TV60 & 165 & 258 & 373 \\
M3-TV0 & 60 & 82 & 109 \\
M3-TV20 & 129 & 198 & 283 \\
M3-TV40 & 165 & 257 & 373 \\
M3-TV60 & 175 & 274 & 398 \\
M4-TV0 & 51 & 67 & 87 \\
M4-TV20 & 111 & 167 & 237 \\
M4-TV40 & 179 & 284 & 413 \\
M4-TV60 & 196 & 309 & 451 \\
\hline
\end{tabular}

\section{CONCLUSION}

Les travaux menés avaient pour objectif d'étudier la possibilité de valoriser les mousses de polyuréthane en construction de substrats pour la croissance de plantes ornementales. Les mousses PU étant peu denses (environ $40 \mathrm{~kg} \cdot \mathrm{m}^{-3}$ ), elles présentent l'avantage d'alléger le substrat. Ainsi, la création d'un substrat adapté à des toitures végétalisées en système extensif (80 à $180 \mathrm{~kg} . \mathrm{m}^{-2}$ ) est envisageable. $D^{\prime}$ un point de vue analytique, l'ajout de mousses au sein d'un compost naturellement riche permet d'atténuer les teneurs de certains éléments nutritifs issus principalement du compost. Cet ajout permet d'obtenir des mélanges proposant une valeur nutritive intéressante pour les plantes, ainsi que des propriétés physiques adaptées pour la croissance végétale. Les résultats de l'expérimentation menée montrent qu'on ne peut pas se passer de la terre végétale pour favoriser la croissance du Ray-grass. Cependant, l'aménagement de toitures terrasses en système extensif doit prendre en compte des problématiques de portance. Ainsi, les substrats les plus adaptés à ce type d'aménagement ne sont pas ceux produisant le plus de biomasse. Cette première étude a permis de sélectionner trois substrats (M3TV20, M4-TV20 et M4-TV40) qui seront testés dans une expérimentation en conditions extérieures et à plus grande échelle. Ce nouvel essai de 2 ans permettra de tester le développement d'autres espèces végétales adaptées aux toitures végétalisées. La mise en place dans des bacs lysimétriques permettra de pouvoir réaliser un suivi analytique des eaux de percolation, afin de vérifier l'innocuité environnementale obtenue après les premières analyses de cette étude.

\section{REMERCIEMENTS}

Ce projet a été financé par l'éco-organisme Ecomobilier (programme Eco-innovation Challenge 2017). Nous souhaitons remercier les membres de la plateforme Phenotic pour leur appui technique, Anaïs Lefilliatre (JCP Environnement) pour son expertise sur la règlementation relative aux toitures végétalisées, et Yvette Barraud-Roussel (Institut Agro RennesAngers, Unité EPHor) pour son soutien technique dans les analyses d'échantillons.

\section{RÉFÉRENCES BIBLIOGRAPHIQUES}

ADIVET, PDE, \& EMB, 2018. Règles professionnelles pour la conception et la réalisation des terrasses et toitures végétalisées, Paris. $84 \mathrm{p}$.

Becker M., Asch F., (2005). Iron toxicity in rice-conditions and management concepts. J. Plant Nutr. Soil Sci, 4, 558573. https://doi.org/10.1002/jpln.200520504

Boujard C., Foray N., Caudron J.-C., 2014. Panorama du marché du polyuréthane et état de l'art de ses techniques de recyclage, Angers, ADEME. $106 \mathrm{p}$.

Damas O, Coulon A., 2016. Créer des sols fertiles : du déchet à la végétalisation urbaine. Chapter 1 : Construire des sols pour végétaliser la ville. $335 \mathrm{p}$. 
Gobat J.-M., Aragno M., Matthey W., 2010. Le sol vivant : bases de pédologie, biologie des sols, Presses polytechniques et universitaires romandes. $817 \mathrm{p}$.

Lê S., Josse J. \& Husson F., (2008). FactoMineR: An R Package for Multivariate Analysis. Journal of Statistical Software, 25, 1-18. https://doi.org/10.18637/iss.v025.i01

Lovett D., Eastop D., (2004). The degradation of polyester polyurethane: preliminary study of 1960 s foam-laminated

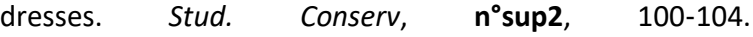
https://doi.org/10.1179/sic.2004.49.s2.022

Pellizzi E., 2012. Étude du vieillissement des mousses de polyuréthane ester et consolidation par les aminoalkylalkoxysilanes, Evry Val d'Essonne, mémoire de doctorat. $208 \mathrm{p}$.

Séré G., Ouvrard S., Schwartz C., Renat JC., Morel JL., (2008). Soil construction: a step for ecological reclamation of derelict lands. J. Soils and Sediments, 8, 130-136. https://doi.org/10.1065/iss2008.03.277

United Nations. (2018) Revision of World Urbanization Prospects Multimedia Library. United Nations Department of Economic and Social Affairs 